\title{
Fractionating polydisperse polyelectrolytes in packed beds of cellulose fibers
}

\author{
Theo G.M. van de Ven ${ }^{1}$, Laura Ciovica ${ }^{2}$ and Nathalie Tufenkji ${ }^{2}$ \\ ${ }^{1}$ Department of Chemistry, Pulp and Paper Research Centre, and ${ }^{2}$ Department of Chemical \\ Engineering, McGill University, Montreal QC, Canada
}

Corresponding author: theo.vandeven@mcgill.ca

\begin{abstract}
Low molecular weight fractions of polydisperse polyelectrolytes can penetrate the porous structure of cellulose fibers, whereas the high molecular weight fraction cannot. The effects of polydispersity on polyelectrolyte adsorption, especially for polyelectrolytes with a very broad size distribution, often encountered in industry, can be very pronounced. Previously we showed that the adsorption rate constant for polyethylene imine (PEI) adsorbing on pulp fibers, as well as the maximum adsorption capacity, roughly increased by a factor of 4 , when the $\mathrm{pH}$ was increased from 6 to 10 . The increase in adsorption capacity was explained by the decrease in the size of a PEI molecule, by almost a factor of two. The increase in rate was explained by the increase in the low molecular weight fraction of PEI which could penetrate the fibers. To explain the results, this low molecular weight fraction should be four times as large at $\mathrm{pH} \mathrm{10,} \mathrm{compared} \mathrm{to} \mathrm{pH}$ 6. Here, we discuss the results of experiments on PEI adsorption in packed beds of cellulose fibers, which confirm this hypothesis.
\end{abstract}

\section{Introduction}

The adsorption of polyelectrolytes on surfaces is important in many areas of science and technology. Examples are polyelectrolytes used as flocculants in papermaking, water purification, polyelectrolytes used as stabilizers on colloidal particles, and polyelectrolytes used in layer-by-layer deposition on particles and surfaces. Here, we study the adsorption behaviour of polyethylene imine (PEI) on cellulose fibers. Many contradictory findings about PEI adsorption on cellulose fibers can be found in the literature. Some papers claim an increase in PEI adsorption with molecular weight [1], others a decrease [2,3]; also some studies show an increase in PEI adsorption with salt concentration [4], others a decrease [3], an effect now well understood: the increase in adsorption with salt is due to screening of charges within the PEI (resulting in a smaller size) and the decrease in adsorption with salt to screening of charges between the PEI and the surface. The result is a maximum in adsorption at intermediate salt concentrations [5]. At high salt concentrations, the bond strength between PEI and cellulose is weakened, leading to a dynamic equilibrium between adsorption and desorption [6]. This equilibrium is likely to depend on molecular weight and thus on polydispersity. Usually large molecular weight polymers are adsorbed preferentially and their desorption rates will be lower [5]. Many years ago, we attempted to reconcile these data [6] and showed that most commercial PEI samples were very polydisperse, which had a tremendous effect on the adsorption behaviour. For instance, it was found that the kinetics of PEI adsorption on cellulose fibers (i.e. the amount of PEI adsorbed as a function of time) was four times larger at $\mathrm{pH} 10$ than at $\mathrm{pH} 6$ and that the maximum adsorption capacity was four times larger as well. The maximum adsorbed amount far exceeded the amount that can be accommodated on the external surface of the fibers, and thus most adsorption 
occurs within the fiber wall, which can only be penetrated by the low molecular weight fraction. When the concentration of PEI is increased during an adsorption experiment, more of this low molecular weight fraction is present and thus more PEI adsorbs. A critical PEI size exists, above which the PEI molecules are too large to enter the pores of the fiber wall, and below which the adsorption increases with molecular weight in the same way as on an external surface [7]. From adsorption experiments with PEI of various sizes and molecular weights, it was found that, at $\mathrm{pH} 10$, the size, $a$, scales as: $a \propto M^{0.39}$, and the maximum adsorbed amount, $\Gamma_{\max }$, as: $\Gamma_{\max } \propto M^{0.26}$ [7]. These results also show that PEI approximately maintains its shape upon adsorption, in which case one expects that:

$$
\Gamma_{\max } \propto \frac{m_{p}}{\pi a^{2}} \propto \frac{M}{M^{0.78}} \propto M^{0.22}
$$

where $m_{\mathrm{p}}$ is the mass of a PEI molecule, proportional to the molecular weight $M$. The exponent 0.22 is close to the experimentally observed value of 0.26 , implying that Eq.1 describes PEI adsorption reasonably well. That PEI approximately maintains its shape upon adsorption has also been shown by AFM imaging of PEI molecules adsorbed on latex beads [8]. This can be readily understood by considering the number of charges of a PEI molecule and the number of surface charges. At $\mathrm{pH} \mathrm{6,a}$ single PEI molecule of $\mathrm{M}_{\mathrm{w}}$ 0.6 MDa contains about 6000 charged groups (protonated amine groups). When adsorbed on a cellulose surface, the surface area in contact with PEI contains about 10 charges. It is clear that this surface charge is insufficient to deform the globular branched PEI molecule to any appreciable extent. At $\mathrm{pH} \mathrm{10,} \mathrm{the} \mathrm{charges} \mathrm{on} \mathrm{a} \mathrm{PEI} \mathrm{molecule} \mathrm{are} \mathrm{an} \mathrm{order} \mathrm{of}$ magnitude smaller, whereas the number of charges on the surface is $4 \mathrm{x}$ smaller (due to the smaller PEI size); thus also here the PEI charges largely dominate the surface charges. The scaling results were obtained on PEI adsorbing on non-porous surfaces, but the results on pulp fibers imply that they hold equally for the adsorption of the low molecular weight fraction. Eq.1 predicts that when the size of a PEI molecule is doubled, this results in a reduction of the maximum adsorption by a factor of 4. This mostly explains the effect of $\mathrm{pH}$ on PEI adsorption, as the size of PEI was found to be $26 \mathrm{~nm}$ at $\mathrm{pH} 10$ and $40 \mathrm{~nm}$ at $\mathrm{pH} 6$ [6]. An additional effect is that PEI molecules adsorbed at $\mathrm{pH} 6$ are less densely packed, because of electrostatic repulsion.

The observed increase in adsorption rate at $\mathrm{pH} 10$ is more puzzling. It was proposed that the low molecular weight fraction that can penetrate the pores in the fiber wall was increased, because increasing the $\mathrm{pH}$ from 6 to 10 results in a shift of the PEI size distribution to smaller values, as shown schematically in Figure 1.

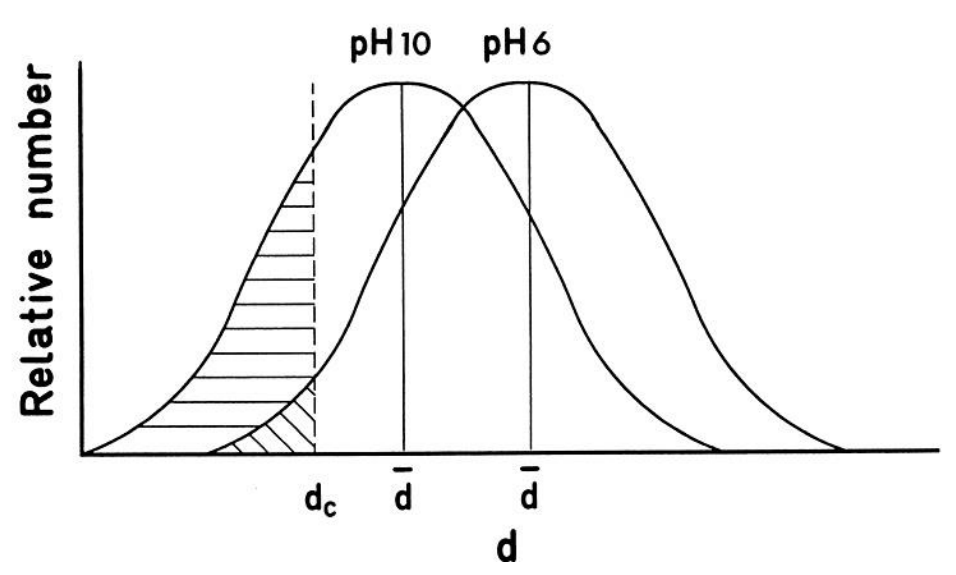

Fig.1. Schematic size distributions of PEI at $\mathrm{pH} 6$ and $\mathrm{pH} 10 ; d_{c}$ denotes the critical diameter of PEI, below which the molecules can freely penetrate the fiber pores (after [6]). 
In order to explain the kinetics of PEI adsorption, it had to be assumed that the fraction of low molecular weight PEI penetrating the pores was four times larger at $\mathrm{pH} 10$ than at $\mathrm{pH} 6$ (cf. Fig.1). This leads to a larger number of PEI molecules that can penetrate the pores and thus in faster kinetics, as the kinetics, i.e. the adsorption rate, is proportional to the number of molecules. No independent evidence of this was provided. Also the observed increase in adsorption capacity cannot be due to the difference in average PEI size, as suggested [6], because the average size is much larger than the pore size, but must be due to a difference in the average size of the low molecular weight fraction, which is able to penetrate the pores. In this paper, we will try to quantify the penetration of low molecular weight PEI in fibers, by studying the adsorption of PEI on fibers packed into a column. It is expected that fibers in the column act as a sink for low molecular weight PEI (which can penetrate the fiber wall), whereas the high molecular weight fraction will either adsorb on the external surface of the fibers or pass freely through the packed bed. As a result, most of the low molecular weight fraction will be removed and only the high molecular weight fraction will pass through. A similar mechanism was proposed for the selective permeation and adsorption of a lowmass component from a polyelectrolyte having a broad molecular mass distribution into a mesoporous substrate [9]. A follow-up work showed that different electrokinetic results were obtained when the low-mass fraction had been removed by dialysis before the adsorption experiment [10]. The present results help to validate that earlier work, in which the proposed mechanism had been demonstrated by an analysis of streaming potential effects but not by breakthrough curves.

The flow through packed beds of fibers has been modeled before, as well as the adsorption of small colloids on the fibers $[11,12]$. Experimental breakthrough curves can be interpreted using these models.

\section{Experimental}

\section{Materials}

As fibers, we used bleached softwood Kraft fibers (Q90, Domtar), which are hollow delignified fibers, with a length of a few $\mathrm{mm}$ (average length $2.37 \mathrm{~mm}$ ) and a diameter of about 25 micron. The thickness of the fiber wall is in the range 3-5 micron. The charge density of the fibers was determined to be $36.5 \mu \mathrm{eq} / \mathrm{g}$ from a conductometric titration. These charges are located throughout the fiber wall and typically the charge groups on the external surface of a fiber are less than $1 \%$ of this value.

As polyelectrolyte, we used polyethylene imine (PEI) (Polymin P, BASF), a highly branched polyelectrolyte containing primary, secondary and tertiary amino groups in the ratio 1:2:1, with a mass average molecular weight $\mathrm{M}_{\mathrm{m}}=6 \times 10^{5}$ and a number average molecular weight of $3.4 \times 10^{4}$. When fully protonated, the charge of PEI is $23.3 \mathrm{meq} / \mathrm{g}$. At $\mathrm{pH} 6$, this charge is reduced to about half. At $\mathrm{pH} 11$, the charge becomes zero [13]. It was used as received, without further purification. The average diameter of PEI, as determined from intrinsic viscosity experiments in the absence of salt, was $40 \mathrm{~nm}$ at $\mathrm{pH} 6$ and $26 \mathrm{~nm}$ at $\mathrm{pH} 10$.

\section{Experimental techniques}


The deposition of PEI on cellulose fibers was studied in packed beds of fibers. Details of this technique can be found in references $[11,12,14]$. The main part consists of a column, in which a bed of cellulose fibers is sandwiched between two screens, and through which one can pass polyelectrolyte and rinsing solutions. The main part of the set-up is shown in Figure 2. The inner diameter of the column is $7.5 \mathrm{~cm}$ and the pad height is typically $4 \mathrm{~cm}$. The column usually contains $19.4 \mathrm{~g}$ of (oven-dried) pulp. The pulp is contained between two 200 mesh papermaking screens and was washed prior to use to remove fines (which interfere with UV-vis measurements) and degassed to remove any air. The flow rate of the PEI solution entering the column was adjusted to 185 $\mathrm{mL} / \mathrm{min}$ by gravity feed.

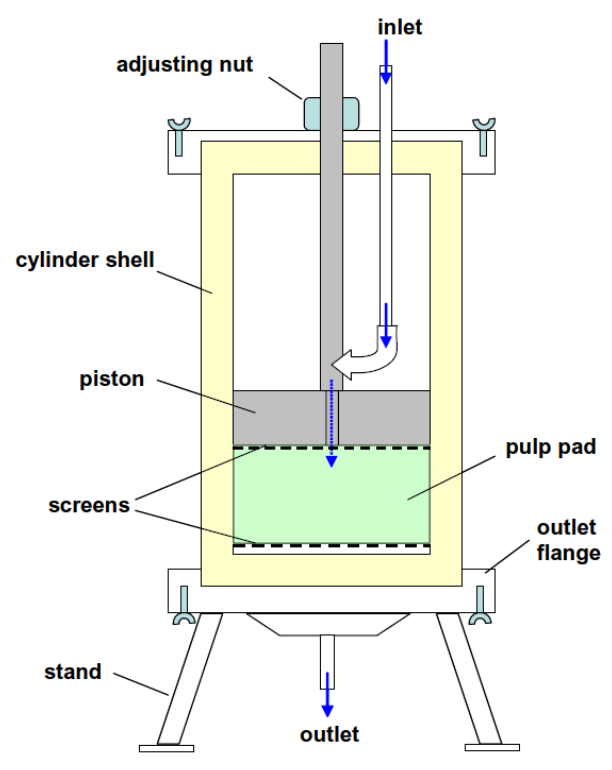

Fig.2. Schematic drawing of packed bed of pulp fibers. Using gravity flow, a PEI suspension can be flown through the column at well-defined velocities. The suspension is collected at the outlet at various time intervals and the concentration of PEI in these samples is determined by a polyelectrolyte titration.

The concentration of PEI in the outlet stream is determined as a function of time, to yield so-called breakthrough curves. The bed is characterized by passing an inert tracer (sucrose) through the column, and measuring its concentration as a function of time in the outlet stream with a UV-vis spectrometer (Varian Cary $1 \mathrm{E}$ UV-Vis) at a wavelength of $191 \mathrm{~nm}$. This yields the residence time of the fluid in the bed $[11,12,14]$.

The packed bed technique is very different from GPC experiments. The pores between the fibers in a packed bed are in the order of $10 \mu \mathrm{m}$, much larger than the sizes of sucrose or PEI molecules. The transport through the bed is determined by the hydrodynamic flow through the bed, coupled to the adsorption and desorption of molecules on the fibers. The packed bed technique is also different from affinity paper chromatography, in which conditions are such that the paper never gets saturated. It is more convenient to think of the packed bed as a large collection of randomly located stagnation point flow (or impinging jet) collectors, which are commonly used in studies of polymer adsorption [15,16]. A molecule passing through the packed bed, encounters more than 1000 such collectors (bed height/fiber diameter) and either adsorbs on one of them or leaves the bed in the exit stream. In fiber beds, adsorption does not only occur in the area around the stagnation point, but all along the fiber and is further complicated by the porous structure of the fiber wall, in which molecules can penetrate.

All packed bed experiments were performed with distilled water and the ionic strength was not altered, other than by changes in $\mathrm{pH}$. 


\section{Polyelectrolyte titration}

To determine the breakthrough curves, samples were taken each minute from the outlet of the column and a polyelectrolyte titration was performed on each sample to determine its PEI concentration. The titration was performed with a Wetrohm 665 Dosimat instrument. The solutions were titrated with the potassium salt of polyvinyl sulfonate (PVSK) (Aldrich) having a molecular weight of $170 \mathrm{kDa}$. As buffer we used potassium hydrogen phthalate (A.C.S. acidimetric standard, Sigma Ultra) to which $1 \mathrm{~mL}$ of o-toluidine blue (Aldrich) is added, which acts as the endpoint indicator of the titration. Details of the titration method can be found in reference [13]. First, a calibration curve was established, which was shown to be linear: $[\mathrm{PEI}]=-0.40+6.96[\mathrm{PVSK}]$, with a regression coefficient of $\mathrm{R}^{2}=0.9976$. Here, $[\mathrm{PEI}]$ is in units of $\mathrm{mg} / \mathrm{L}$ and $[\mathrm{PVSK}]$ in $\mathrm{mL}$. For a sample with an unknown PEI concentration, the amount of PVSK required to cause a color change in o-toluidine blue was determined by titration, from which the PEI concentration can be found from the calibration curve.

\section{Results and Discussion}

A typical PEI breakthrough curve is shown in Figure 3, which shows the PEI concentration, $n$, in the outlet stream, normalized by the concentration of the inlet stream, $n_{\mathrm{o}}$, as a function of dimensionless time, $\tau$, defied as $\tau=t / t_{\text {res, }}, t_{\text {res }}$ being the average residence time of a PEI solution in the bed. In these experiments, $t_{\text {res }}$ is on the order of one minute, determined from the breakthrough curve of sucrose and defined as the time at which the dimensionless sucrose concentration in the outlet reaches 0.5. The value varies slightly from bed to bed, as not all beds are exactly the same. Fig. 3 also shows a repeat experiment, showing the reproducibility of the results.

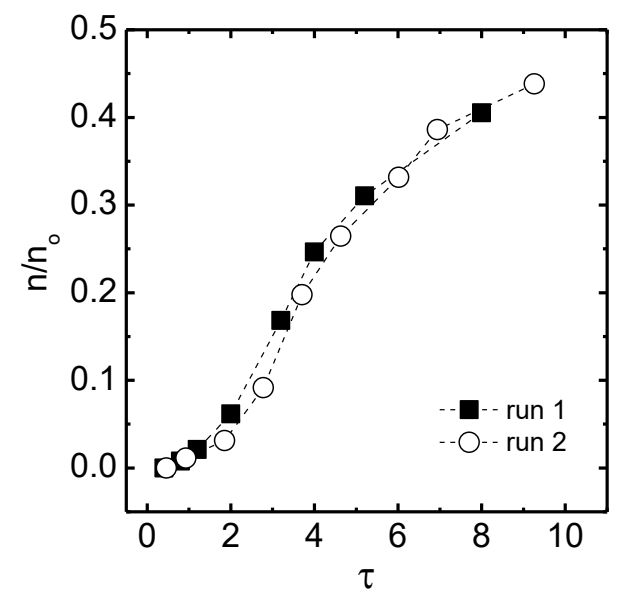

Fig.3. Breakthrough curves for a PEI solution of concentration $9 \mathrm{mg} / \mathrm{L}$ at $\mathrm{pH} 6$ and flow rate $185 \mathrm{~mL} / \mathrm{min}$, through a packed bed of cellulose fibers.

Results for various concentrations of PEI at $\mathrm{pH}$ 6, as well as for an inert tracer (sucrose), are shown in Figure 4. They show that at low PEI concentrations, all PEI adsorbs on the fibers and that with increasing PEI concentration, the breakthrough occurs earlier. 


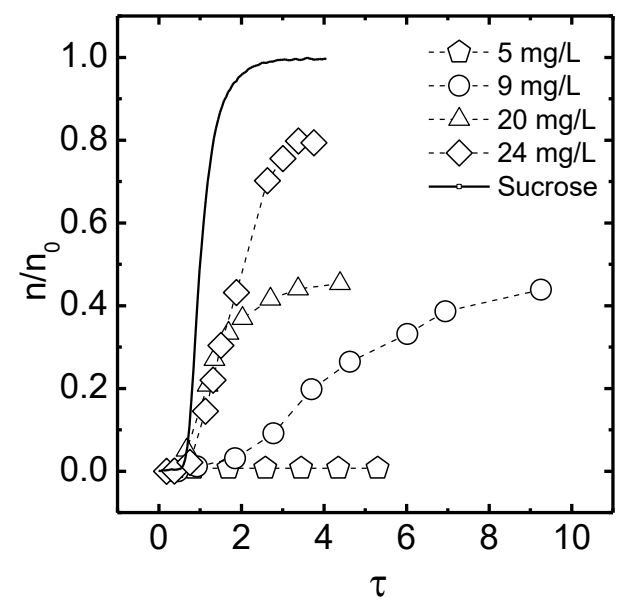

Fig.4. Breakthrough curves for PEI at various inlet concentrations (indicated in the figure), at $p H 6$. Also shown is the breakthrough curve for an inert tracer (sucrose).

Similar results for experiments conducted at $\mathrm{pH} 10$ are shown in Figure 5. It can be seen that much more PEI adsorbs at $\mathrm{pH} 10$ than at $\mathrm{pH}$ 6, as can be deduced from the PEI concentrations in the outlet stream which are much lower.

Fig.5. Breakthrough curves for PEI at various inlet concentrations (indicated in the figure), at pH 10. Also shown is the breakthrough curve for an inert tracer (sucrose).

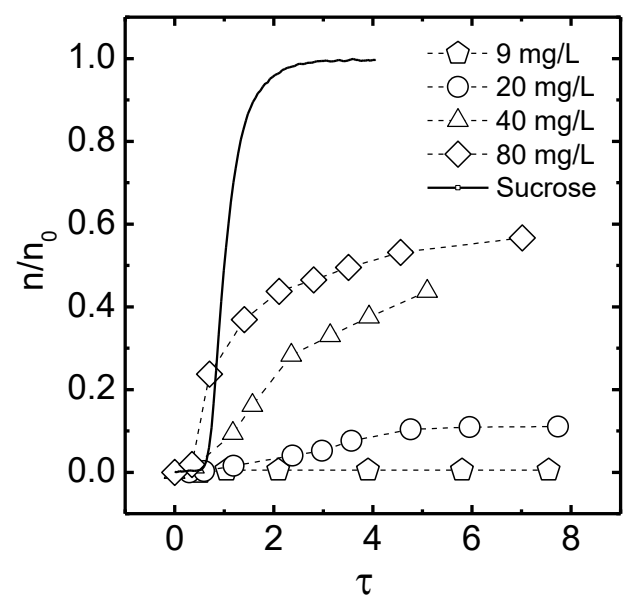

The breakthrough curve for a high inlet concentration of $80 \mathrm{mg} / \mathrm{L}$ is especially interesting. Initially, the breakthrough is very similar to that of an inert tracer. The same was observed for higher PEI concentrations (not shown). This implies that at high PEI concentrations, the external surface of the fibers gets covered very fast by PEI and that the adsorbed amount is small compared to the inlet concentration. Based on the volume of the packed bed and the amount of fibers in it, one can estimate that the volume of liquid between the fibers is about $150 \mathrm{~mL}$, which at $80 \mathrm{mg} / \mathrm{L}$, contains about $12 \mathrm{mg}$ of PEI. It was estimated in a previous study [4], that at $\mathrm{pH} 6$ about $10 \%$ of the PEI can penetrate the pores and that the adsorption on the external surface is $0.14 \mathrm{mg} / \mathrm{g}$. At $\mathrm{pH} 10$, this amount is expected to increase to about $0.6 \mathrm{mg} / \mathrm{g}$. As there is about $20 \mathrm{~g}$ of fiber in the bed, the adsorption on the external surface at $\mathrm{pH} 10$ is about $1.2 \mathrm{mg}$, or about $10 \%$ of the PEI in the bed. Thus, most PEI (about 90\%) can pass through the bed and the breakthrough resembles that of an inert tracer. However, the maximum PEI adsorption at $\mathrm{pH} 10$ on similar cellulose fibers is reported as $22 \mathrm{mg} / \mathrm{g}$ [8] and most of this is adsorbed inside the fiber wall. The kinetics of adsorption of the 
low molecular weight fraction of PEI is much slower, and this is reflected in the breakthrough curves, which take longer to reach a plateau.

The breakthrough curve for $[\mathrm{PEI}]=80 \mathrm{mg} / \mathrm{L}$ slowly approaches an apparent plateau of about 0.6 (Figure 5). This implies that $40 \%$ of the PEI that enters the packed bed is adsorbed continuously. Eventually the bed must saturate, but this takes a long time. At a flow rate of 185 $\mathrm{mL} / \mathrm{min}$ and $32 \mathrm{mg} / \mathrm{L}$ adsorbed, about $6 \mathrm{mg}$ is adsorbed per minute, whereas the maximum adsorption capacity for $19.4 \mathrm{~g}$ of fibers is about $427 \mathrm{mg}$. So it takes well over an hour to saturate the bed, which is much longer than the time of an experiment.

If this interpretation is correct, it follows that PEI solutions, passing through the bed at times for which the breakthrough curve has reached an apparent plateau, should contain very little of the low molecular weight material. To test this we collected such PEI, which we refer to as fractionated PEI, and passed it through a newly made column, expecting to see breakthrough curves reaching $100 \%$ breakthrough.

PEI solutions of $80 \mathrm{mg} / \mathrm{L}$ at $\mathrm{pH} 10$ were passed through a packed bed of fibers and PEI solutions were collected at the outlet of the column for time periods greater than 15 minutes. The $\mathrm{pH}$ was adjusted to 6 and the fractionated PEI was passed through a new packed bed. The reason the PEI was fractionated at $\mathrm{pH} 10$, instead of 6 , is that at $\mathrm{pH} 10$ the fraction that can penetrate the fibers is larger, leading to a better fractionation. The results of this experiment are shown in Figure 6.

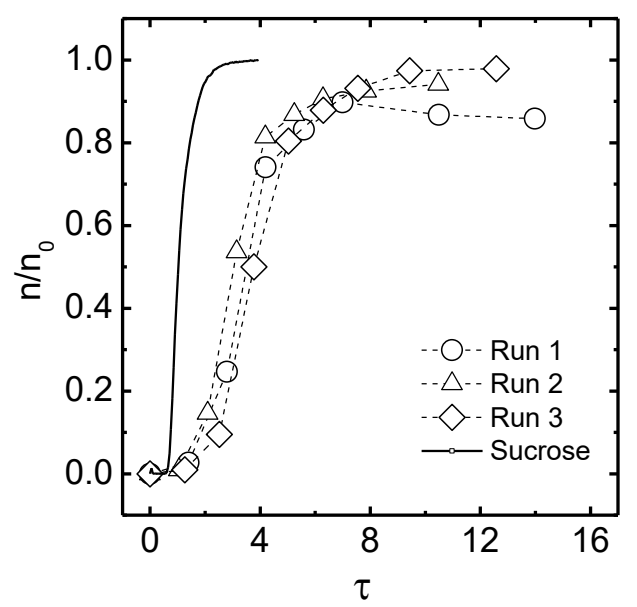

Fig.6. Breakthrough curves for fractionated PEI at an inlet concentration of $4.25 \mathrm{mg} / \mathrm{L}$ at $\mathrm{pH}$ 6. Also shown is the breakthrough curve for an inert tracer (sucrose).

It can be seen that the breakthrough curves are very different from the ones of the non-fractionated samples, shown in Figure 4, where for a similar PEI concentration $(5 \mathrm{mg} / \mathrm{L})$ no measurable breakthrough occurred. In Figure 6, the breakthrough curves reach nearly 100\% breakthrough $\left(n / n_{0}\right.$ $=1$ ). The shape of these breakthrough curves is in agreement with the adsorption of high molecular weight PEI. For a PEI inlet concentration of $4.25 \mathrm{mg} / \mathrm{L}$ and a maximum adsorption of $0.14 \mathrm{mg} / \mathrm{g}$, about $0.3 \mathrm{mg} \mathrm{PEI} / \mathrm{min}$ is passing through the bed, which has a maximum adsorption capacity of about $2.8 \mathrm{mg}$. Hence, it takes close to 10 minutes $(2.8 / 0.3)$ to reach saturation, in good agreement with the data in Figure 6.

From the area between the breakthrough curves for an inert tracer and an adsorbing molecule, one can determine the maximum adsorption capacity. For the data in Figure 6, one cannot obtain an accurate estimate, because of the uncertainty how the plateau is reached. 
Nevertheless, the dimensionless area between these curves is about 2.7, which for a flow rate of 185 $\mathrm{mL} / \mathrm{min}$, an inlet concentration of $4.25 \mathrm{mg} / \mathrm{L}$, and a residence time of one minute, corresponds to $2.1 \mathrm{mg} / 19.4 \mathrm{~g}$ of fiber, or $0.11 \mathrm{mg} / \mathrm{g}$, which is close to the $0.14 \mathrm{mg}$ value mentioned above.

The observation (Fig. 4) that no measurable PEI adsorption occurs within 5 minutes for the non-fractionated sample at a $[\mathrm{PEI}]=5 \mathrm{mg} / \mathrm{L}$, can be readily understood: At these conditions, about $4.6 \mathrm{mg}$ PEI passes through the bed. This amount includes the $40 \%$ removed that can be removed by fractionation. Thus, compared to the data in Figure 6, only $60 \%$ is high molecular weight PEI, or $2.8 \mathrm{mg} / \mathrm{g}$. This is close to the maximum adsorption capacity of $20 \mathrm{~g}$ fiber. Thus all high molecular weight can adsorb, as does the low molecular weight fraction. For longer times or higher PEI concentrations, more PEI passes through the bed than can adsorb, and thus PEI is detected in the outlet stream.

\section{Conclusions}

We have shown that polydispersity in molecular weight has a tremendous effect on the adsorption behaviour of PEI. The size of a PEI molecule depends on $\mathrm{pH}$ and thus the fraction of PEI that can penetrate the pores in cellulose fibers depends on $\mathrm{pH}$ as well. Passing PEI through a bed of fibers removes the low molecular weight fraction, because fibers act as a sink for the small PEI molecules. Breakthrough curves for fractionated PEI passed through packed beds of fibers are very different from those for non-fractionated PEI solutions. We have shown earlier [7] that the adsorption behaviour of PEI on porous glass is very similar as that on fibers. Thus, in principle, monodisperse fractions of PEI can be obtained using beds of porous glass beads with different pore sizes.

\section{Acknowledgement}

The authors would like to acknowledge financial support from a NSERC/FPInnovations Industrial Research Chair in "Colloid and Papermaking Chemistry". The help of Ivan Rafael Quevedo with the figures is also acknowledged. 


\section{References}

1. D. Horn and J. Melzer, Trans. 6 ${ }^{\text {th }}$ Fund. Research Symp., "Fibre-water interactions in papermaking”, Vol.1, p.19, Oxford Univ. Press, Oxford, 1979

2. G.G. Allan and W.M. Rief, Svensk Paperstidn. 74(2), 25 (1971)

3. B. Alince, Cell. Chem. Techn. 8(6), 573 (1974)

4. J. Petlicki and T.G.M. van de Ven, Colloids \& Surfaces A, 83, 9 (1994)

5. G.J. Fleer, M.A. Cohen Stuart, J.M.H.M. Scheutjens, T. Cosgrove and B. Vincent, "Polymers at interfaces", Chapman \& Hall, London, 1993, pp. 272 and 347-348.

6. B. Alince, A. Vanerek and T.G.M. van de Ven, Ber. Bunsenges. Phys.Chem. 100, 954 (1996)

7. B. Alince and T.G.M. van de Ven, Trans. $11^{\text {th }}$ Fund. Research Symp., "The fundamentals of papermaking materials", Vol.2. p. 771, Pira Intern. Press, 1997

8. S. Akari, W. Schrepp and D. Horn, "Imaging single polyethylenimine polymers adsorbed on negatively charged latex spheres by chemical force microscopy”, Langmuir 12, 857 (1996)

9 M.A. Hubbe, O.J. Rojas, L.A. Lucia and T.M. Jung, "Consequences of the nanoporosity of cellulosic fibers on their streaming potential and their interactions with cationic polyelectrolytes," Cellulose 14(6), 655-671 (2007).

10. N. Wu, M.A. Hubbe, O.J. Rojas, and S. Park, "Permeation of a cationic polyelectrolyte into mesoporous silica. Part 1. Factors affecting changes in streaming potential," Colloids and Surfaces A 364(1-3), 1-6 (2010).

11. M. Al-Jabari, A.R.P. van Heiningen and T.G.M. van de Ven, J. Pulp Paper Sci.,20(9), J249 (1994)

12. M. Al-Jabari, A.R.P. van Heiningen and T.G.M. van de Ven, J. Pulp Paper Sci., 20(10) J289 (1994)

13. D. Horn, Progr. Colloid \& Polymer Sci. 65, 251 (1978)

14. O. Ali, PhD thesis, Dept. Chem. Eng. McGill University, 1989

15. T.G.M. van de Ven and S.J. Kelemen, Characterizing Polymers with an Impinging Jet J. Colloid Interf. Sci. 181, 118-123 (1996)

16. J.C. Dijt, M.A. Cohen Stuart, J.E. Hofman and G.J. Fleer, Kinetics of polymer adsorption in stagnation point flow Colloids and Surfaces Volume 51, 141-158 1990, 